\title{
Value of DNA analysis in addition to cytological testing in the diagnosis of malignant pleural effusions
}

Felipe Rodríguez de Castro, Teresa Molero, Orlando Acosta, Gabriel Julià-Serdà, José Caminero, Pedro Cabrera, Teresa Carrillo

\begin{abstract}
Background - Aneuploidy appears to be a highly specific marker for cancer, and measurement of cellular DNA content by flow cytometry is rapid and reliable. This study was undertaken to determine if the addition of DNA analysis improved the sensitivity of cytological diagnosis of malignancy in pleural fluid.
\end{abstract}

Methods - Pleural effusions from 92 patients were studied by cytological examination and flow cytometry.

Results - In 41 patients the final diagnosis was malignancy, there were 40 cases of benign effusions including 22 with pleural tuberculosis, and in the remaining 11 patients with biopsy proven cancer the presence of malignant cells was not found by cytological and histological means in the pleural fluid. Aneuploidy and cytological malignancy were found in 14 samples. There were seven cases with abnormal flow cytometry and negative cytological results. In 12 patients the cytological test results were positive but DNA analysis was normal. Thirty six samples of fluid were both diploid and cytologically negative. Of the 22 tuberculous effusions seven contained aneuploid cells. The sensitivity of DNA and cytological analysis was $51 \cdot 2 \%$ and $63 \cdot 4 \%$, respectively. The specificity of DNA analysis was $74 \cdot 5 \%$.

Conclusions - DNA analysis of cells in malignant pleural effusions is both less sensitive and specific than the cytological diagnosis. Flow cytometric analysis is not recommended for routine use in the diagnosis of pleural effusions.

(Thorax 1994;49:692-694)

Malignant disease causes, or is associated with, up to $77 \%$ of all pleural effusions. ${ }^{1}$ Of particular prognostic and therapeutic significance is the presence or absence of malignant cells, which may be found either on cytological examination of pleural fluid or by histological examination of pleural tissue. In general, cytological examination of pleural fluid establishes the diagnosis more frequently than pleural biopsy, with positive rates of $25 \%$ to $87 \% .^{23}$ This variability depends mainly on the type of malignancy and on the skill of the cytologist. Moreover, in many patients with proven malignancy pleural effusions are caused by neoplastic involvement of the mediastinal lymph nodes rather than by direct invasion of the parietal pleura. ${ }^{4}$ Additional non-invasive diagnostic techniques would therefore be useful to identify tumour cells in pleural fluid.

Malignant tumours generally exhibit abnormal amounts of DNA, and measurement of DNA content by flow cytometry has been used to complement cytological or histological diagnosis. ${ }^{5}$ However, details of the accuracy of flow cytometry for differentiating between benign and malignant pleural effusions remain scanty. ${ }^{67}$ We have prospectively studied pleural effusions using both conventional cytopathology and flow cytometry to determine if the addition of DNA analysis improves the sensitivity of cytological diagnosis.

\section{Methods}

PATIENTS AND SAMPLES

Between December 1991 and April 199392 samples of pleural fluid obtained from consecutive unselected patients undergoing a therapeutic or diagnostic thoracocentesis were examined. According to the final diagnosis achieved the effusions were divided into three groups: group I - malignant effusions where a diagnosis of malignancy was made by cytological examination of pleural fluid, or by histological examination of parietal pleural biopsy samples obtained from a blind biopsy or thoracoscopy, or both; group II - benign effusions that included 22 with tuberculosis, four parapneumonic effusions, three cases of congestive heart failure, two cases of collagen disease, one nephrotic syndrome, one cirrhosis, and seven with unidentified causes; group III - effusions associated with known malignancy where cytological examination of the pleural fluid and histological testing of biopsy samples obtained by both pleural biopsy and thoracoscopy could not demonstrate metastases.

CYTOLOGICAL TESTING AND FLOW CYTOMETRY The samples were obtained from fresh pleural fluid and sent for conventional diagnostic cytological testing which was performed on cells stained with a Papanicolau stain and interpreted by a cytopathologist. An aliquot of 20 $50 \mathrm{ml}$ of pleural fluid was also sent for flow cytometric analysis and processed within four hours. The samples were centrifuged at $350 \mathrm{~g}$ for five minutes, the supernatant was discarded, and the cell pellet was resuspended with phosphate buffered saline (PBS). The 
suspension was filtered through a $50 \mu \mathrm{m}$ pore size nylon mesh (Pall Utipor SQ 40S Pall, Spain) to remove large clumps, and was then washed in PBS and the concentration adjusted to $1 \times 10^{8}$ nuclei per sample. Samples containing red blood cells were lysed before being processed.

Cell suspensions were frozen by adding $1.5 \mathrm{ml}$ citrate buffer solution containing sucrose and dimethyl sulphoxide, stored at $-80^{\circ} \mathrm{C}$, and melted in a water bath at $37^{\circ} \mathrm{C}$. At the same time peripheral blood from healthy volunteers was processed and added for analysis as an external control to provide a reference point for determining the DNA index within a given sample. Once melted, the samples were centrifuged for five minutes at $350 \mathrm{~g}$ at room temperature and the supernatant was aspirated. The samples were stained as described elsewhere. ${ }^{8}$

Samples were run in the flow cytometer for between 15 minutes and three hours after the addition of propidium iodide solution using a Facscan Analyzer flow cytometer (Becton Dickinson, Mountain View, California, USA). A minimum of 10000 nuclei were collected in each DNA histogram.

The coefficient of variation (CV) of the diploid peaks was always below $5 \%$, otherwise samples were excluded from the series. The percentage of the $S$ phase fraction was calculated using the "broadened rectangle" mathematical model (Hewlett-Packard software). ${ }^{9}$ Mixed solutions of pleural fluid and peripheral blood nuclei were run when the pleural effusion contained $100 \%$ tumour cells. DNA abnormalities were expressed quantitatively as a DNA index which is the ratio of the positions on the abscissa of an abnormal peak to that of the normal diploid cells. A population of cells with DNA content deviating less than $10 \%$ from the standard was considered to be diploid, thus the value for diploid nuclei was $1 \pm 0 \cdot 1$. Lower or higher DNA indices were considered hypodiploid or hyperdiploid, respectively. A pseudodiploid peak was a DNA histogram with a DNA index of $0.80-0.90$ or $1 \cdot 10-1 \cdot 24$. Aneuploidy was diagnosed when more than $10 \%$ of the cells were aneuploid. Neither a "shoulder" of the $G_{1}$ peak nor a wide $\mathrm{G}_{1}$ peak was considered aneuploid.

\section{STATISTICAL ANALYSIS}

Data are presented as mean (SE) values. Continuous data were compared between groups using the $t$ test. The Pearson correlation coefficient was used to examine relationships between two variables, $p$ values $<0.05$ being considered statistically significant.

Test sensitivity was calculated as test positive/total positive (\%), and specificity as test negative/total negative $(\%)$.

\section{Results}

Of the 92 patients entered 62 were men and the mean age was $56 \cdot 7(1.95)$ years (range 16-85).

Forty one patients had effusions due to malignant disease (group I); 20 of these had a
Flow cytometry and cytological diagnosis in malignant effusions

\begin{tabular}{lll}
\hline & \multicolumn{2}{l}{ Cytological examination } \\
\cline { 2 - 3 } Flow cytometry & Positive & Negative \\
\hline Positive & 14 & 7 \\
Negative & 12 & 8 \\
\hline
\end{tabular}

normal DNA histogram and the remaining 21 had pleural effusions in which aneuploid cells were found. There was agreement between the results of flow cytometric analysis and cytological examination in eight negative samples and 14 positive samples. There were seven cases in which flow cytometry was abnormal but cytological results were negative. In 12 patients the cytological results were positive for malignancy but DNA analysis was normal (table). The diagnosis of a malignant effusion in all 41 patients in this group was confirmed by histological examination of pleural tissue or highly specific procedures such as immunostaining in two cases of acute myeloid leukaemia.

There were 40 patients with benign effusions (group II), including 22 cases of pleural tuberculosis. Ten were aneuploid and the remaining 30 were diploid. Seven of the 10 aneuploid samples were from the cases of tuberculosis, one had cirrhosis with ascites, and two remained undiagnosed. In both these latter cases the effusions resolved spontaneously during follow up.

Eleven patients had a history of malignant disease with incidental pleural effusions thought to be not directly due to cancer (group III), with no malignant cells on cytological or histological examination. Eight of these effusions were negative to DNA analysis. In three cases flow cytometry was abnormal and the associated tumours detected were bronchogenic squamous cell, cervical squamous cell carcinoma, and prostatic adenocarcinoma. The case of bronchogenic carcinoma was resected and its postoperative staging was $\mathrm{T}_{2} \mathrm{~N}_{2} \mathrm{M}_{0}$.

As malignant effusions may be characterised by abnormal proliferative activity we calculated the percentage of cells in the $S$ phase and found a significantly higher $S$ phase fraction in malignant pleural effusions than in benign ones $(7.5(1.45) v 1.99(0.24) ; \mathrm{p}<0.01)$. Similarly, a correlation was seen between the $S$ phase fraction and DNA index $(r=0.57$; $\mathrm{p}<0.05)$.

The sensitivity of DNA analysis was $51 \cdot 2 \%$, a lower figure than that obtained from cytological analysis $(63.4 \%)$. Considering both methods together, the sensitivity was $80 \cdot 4 \%$. In contrast, the specificity of DNA analysis was only $74.5 \%$ compared with $100 \%$ for conventional cytological examination. This low specificity was due mainly to the large number of cases with pleural tuberculosis and aneuploid cells (seven of 22).

\section{Discussion}

Neoplastic lesions often undergo chromosomal aberrations resulting in the appearance of non- 
diploid clones within the tumour cell population. The finding of aneuploidy is therefore highly suggestive of malignancy with pronounced karyotype abnormalities. Flow cytometry allows rapid quantitative measurement of nuclear DNA and could therefore be used as a tool for differentiating between benign and malignant cells.

In our series DNA analysis was falsely negative in 20 cases, a sensitivity of $51 \cdot 2 \%$. Conventional cytological examination had a sensitivity of $63.4 \%$. When both techniques were combined the sensitivity achieved $80 \cdot 4 \%$. There is only one study that reports aneuploid DNA patterns in all malignant effusions studied, although some of them showed minimal deviation of DNA diploid standard. ${ }^{7}$ All other communications, dealing with different body fluids, have shown sensitivities between $30 \%$ and $88 \% .^{610-14}$ These results may be due to: (1) a lack of sensitivity of flow cytometric analysis which cannot detect karyotype abnormalities belonging to one or two chromosomes; (2) some cancer cells being diploid as occurs in approximately $20 \%$ of all solid tumours; and (3) pleural effusions, which contain a small number of malignant cells, may require a larger sample than the one studied to assess aneuploidy.

With the exception of Hedley et $a l^{10}$ and Schneller et $a l^{13}$ all other authors have found some false positive results with a specificity ranging from $86 \%$ to $96 \% .{ }^{67111215}$ However, the definition of aneuploidy has not always been uniform. In our study 10 samples were considered false positive because of abnormal DNA content with no evidence of malignancy. It is noteworthy that almost one third of the tuberculous effusions showed DNA aneuploidy, although five out of seven had a pseudodiploid pattern which is an unreliable marker of malignancy. The mechanisms by which Mycobacterium tuberculosis produces DNA aneuploidy are not clear. T lymphocytes specifically sensitised to tuberculous protein might be responsible for the discrete changes in DNA ploidy patterns. The kinetics of PPD reactive $\mathrm{T}$ lymphocytes in the pleural space may influence the finding of aneuploidy in only some patients. The large number of cases of tuberculosis in our series may explain the low specificity observed.

In conclusion, since DNA analysis of cells in malignant pleural effusions was both less sensitive and specific than cytological diagnosis, flow cytometric analysis is not recommended for routine use.

1 Light RW. Pleural diseases. 2nd ed. Philadelphia: Lea and Febiger, 1990;97-115.

2 Melamed MR. The cytological presentation of malignant lymphomas and related diseases in effusions. Cancer lymphomas and

3 Grunze $H$. The comparative diagnostic accuracy, efficiency and specificity of cytologic techniques used in the diagnosis of malignant neoplasm in serous effusions of the pleural and pericardial cavities. Acta Cytol 1964;8:15064.

4 Meyer PC. Metastatic carcinoma of the pleura. Thorax 1966;21:437-43.

5 Barlogie B, Raber M, Schumann J, Johnson TS, Drewinko $\mathrm{B}$, Swartzendruber DE, et al. Flow cytometry in clinical cancer research. Cancer Res 1983;43:3982-97.

6 Unger KM, Raber M, Bedrossian CWM, Stein DA, Barlogie B. Analysis of pleural effusions using automated flow cytometry. Cancer 1983;52:873-7.

7 Evans DA, Thornthwaite JT, Ng ABP, Sugarbaker EV. DNA flow cytometry of pleural effusions: comparison with pathology for the diagnosis of malignancy. Anal Quant Cytol 1983;5:19-27.

8 Rodriguez de Castro F, Molero T, Diaz F, Caminero JA, Cabrera P, Carrillo T. Contenido de DNA nuclear en muestras de carcinoma broncogenico obtenidas mediante fibrobroncoscopia. Arch Bronconeumol 1992;28:161-4

9 Baisch H. A comparison of mathematical methods for the analysis of DNA histograms obtained by flow cytometry. Cell Tissue Kinetics 1982;15:235-49.

10 Hedley DW, Phillips J, Rugg CA, Taylor IW. Measurement of cellular DNA content as an adjunct to diagnostic cytology in malignant effusions. Eur $\mathcal{F}$ Cancer Clin Oncol cytology in malign

11 Stonesifer KJ, Xiang J, Wilkinson EJ, Benson NA, Braylan RC. Flow cytometric analysis and cytopathology of body cavity fluids. Acta Cytol 1987;31:125-30.

12 Katz RL, Johnson TS, Williamson KD. Comparison of cytologic and acridine-orange flow cytometric detection of malignant cells in human body cavity fluids. Anal Quant Cytol Histol 1985;7:227-35.

13 Schneller J, Eppich E, Greenebaum E, Elequin F, Sherman $A$, Wersto R, et al. Flow cytometry and Feulgen cytophotometry in evaluation of effusions. Cancer 1987;59:130713.

14 Pinto MM. DNA analysis of malignant effusions. Comparison with cytologic diagnosis and carcinoembryonic antiison with cytologic diagnosis and carcinoembryonic an

15 Hostmark J, Vigander T, Skaarland E. Characterization of pleural effusions by flow-cytometric DNA analysis. Eur $\mathcal{F}$ Respir Dis 1985;66:315-9. 